\title{
Membrane Protein Biosynthesis at the Endoplasmic Reticulum
}

\author{
Rebecca Voorhees
}

California Institute of Technology, Pasadena, California, United States

Secreted and integral membrane proteins make up $\sim 30 \%$ of the eukaryotic proteome. This family of proteins is essential for a range of cellular functions including intracellular trafficking, cell signaling, and the transport of molecules across the membrane. Defects in membrane protein maturation underlie numerous protein misfolding diseases, and more than half of all therapeutic drugs bind a membrane protein target. The essential roles of these proteins, as well as the consequences of their failed maturation, underscore the physiologic importance of understanding the molecular details of membrane protein biogenesis.

Both secreted and integral membrane proteins contain one or more hydrophobic segments that must be targeted and inserted into the lipid bilayer. In eukaryotes, the majority of membrane proteins are targeted co-translationally to the endoplasmic reticulum (ER) by the signal recognition particle (SRP), and then translocated or inserted into the membrane by the universally conserved Sec61 channel. Substrates that cannot use this co-translational pathway, either because they are too small or contain only a single Cterminal transmembrane helix (i.e. tail-anchored proteins), instead rely on post-translational insertion by either WRB/CAML or the ER membrane protein complex (EMC).

Here we present a series of structures using single particle cryo-EM that trace the path of a hydrophobic nascent chain from its synthesis in the cytosol to its insertion at the membrane. These include structures of SRP bound to the ribosome both before and after substrate engagement. As well as structures of the mammalian ribosome and the Sec61 translocation channel in several functional states. Functional and structural insights into post-translational membrane targeting elucidate several evolutionarily conserved strategies for membrane protein insertion across the lipid bilayer. Together these structures suggest how exposure of a hydrophobic signal is minimized during membrane targeting, while also ensuring the selective recognition and insertion of proteins at the ER. Defining the molecular details of membrane protein biogenesis not only contributes to our understanding of this fundamental biological process, but will also provide insight into how failures in this pathway can lead to disease.

References

Voorhees, R.M., Hegde, R.S. (2016) "Toward a structural understanding of co-translational protein translocation." Curr. Opin. Cell Biol., 41: 91-9. 